\title{
Micro-macro analysis of steel sheet behaviour in finite element simulations. Application to deep-drawing process
}

\author{
L. Duchêne, A. Godinas, S. Cescotto and A.M. Habraken \\ Department of Structures, University of Liège, \\ Chemin des Chevreuils 1, 4000 Liège, Belgium \\ l.duchene@ulg.ac.be
}

\begin{abstract}
This paper presents a constitutive law based on Taylor's model implemented in our non-linear finite element code LAGAMINE. The yield locus is only locally described and a particular interpolation method has been developed. This local yield locus model uses a discrete representation of the material's texture. The interpolation method is presented and a deep-drawing application is simulated in order to show up the influence of the texture evolution during forming processes.
\end{abstract}

\section{STATEMENT OF THE PROBLEM}

The objective of our research is to integrate the influence of the material's texture into a finite element code. The constitutive law describing the mechanical behaviour of the studied sample is based on a microscopic approach. The law computation takes place on the crystallographic level. A large number of crystals must be used to represent correctly the global behaviour. The micro-macro transition links the global behaviour to the crystallographic results. The full constraint Taylor's model is used for the computation of the microscopic behaviour of each crystal and for the micro-macro transition. Unfortunately, this model does not lead to a general law with a mathematical formulation of the yield locus. Only one point of the yield locus corresponding to a particular strain rate direction can be computed.

The "direct Taylor's model" assumes that one macroscopic stress results from the average of the microscopic stresses related to each crystal belonging to a set of representative crystals. The computation of the mechanical behaviour involves a large number of crystals and must be repeated for each integration point of the finite element model, for each iteration of each time step. So, such a micro-macro approach consumes large computation time and seems practically not usable.

However, using different simplified approaches, various constitutive laws based on texture analysis have been implemented in the non-linear finite element code LAGAMINE. Our first step in the integration of the texture effects has been the use of a $6^{\text {th }}$ order series yield locus defined by a least square fitting on a large number of points (typically 70300) in the deviatoric stress space (see [4]). Those points were calculated by Taylor's model based on an assumed constant texture of the material. This fitting is performed once, outside the FEM code. It provides 210 coefficients to describe the whole yield locus. This method, i.e. a global description of the yield locus, is actually used in the FEM code.

Unfortunately, taking into account the texture evolution effects with this yield locus would imply the computation of the 210 coefficients of the $6^{\text {th }}$ order series for each integration point, each time a texture updating is necessary. This would require an impressive amount of computation and memory storage (210 coefficients for each integration point) which is only partially useful as generally the stress state remains in a local zone of the yield locus. So, two new approaches, where the whole yield locus is unknown, have been investigated.

In the first case, some points in the interesting part of the yield locus are computed with Taylor's model. This local zone of the yield locus is then represented by a set of hyperplanes which are planes defined in the five-dimensional deviatoric stress space. These planes being fitted on Taylor's points.

As it has been shown in [2], the yield locus discontinuities bred by this very simple interpolation 
method give rise to convergence problems in the finite element code. That is the reason why a second method has been developed. For that second approach, no yield locus is defined and a direct stress-strain interpolation between Taylor's points is achieved. In this case, the yield stress continuity conditions are fulfilled but, as there is no yield locus formulation, a particular stress integration scheme has to be used.

Both interpolation methods allow us an important computation time reduction with respect to the “direct Taylor's model” application. Taylor's model is only used to compute some points in order to achieve the interpolation. These points must be computed in two cases:

- When the current part of the yield locus does not content anymore the new stress state and that a new local zone of the yield locus is required.

- When the plastic strains significantly deform the material and induce changes in the crystallographic orientations, i.e. when the texture evolves. Indeed, the corresponding mechanical behaviour of the material would no more be correctly represented by the old points. A texture updating must take place.

The part yield locus approach presented in this paper can be placed between the microscopic approach (accurate but very slow) and the global yield locus approach (fast but inaccurate and especially not adapted for texture updating).

This paper describes the stress-strain interpolation method; interested readers can refer to [5] and [2] for the $6^{\text {th }}$ order and the hyperplanes method. The influence of the texture updating during a forming process has been highlighted by a deep-drawing simulation.

\section{STRESS-STRAIN INTERPOLATION}

\subsection{Local description of a scaled yield locus.}

This model is particular in the sense that it does not use a yield locus formulation neither for the interpolation nor in the stress integration scheme.

We use a linear stress-strain interpolation described by Equation 1.

$$
\underline{\sigma}=\tau \cdot \underline{\underline{C}} \bullet \underline{u}
$$

In this equation, $\underline{\sigma}$ is a 5-D vector containing the deviatoric part of the stress; the hydrostatic part being elastically computed according to Hooke's law. The 5-D vector $\underline{u}$ is the deviatoric plastic strain rate direction; it is a unit vector. $\tau$ is a scalar describing the work hardening according to the exponential relationship of Equation 2 where the strength coefficient $K$, the offset $\Gamma^{0}$ and the hardening exponent $n$ are material parameters fitted to experimental data and $\Gamma$ is the polycrystal induced slip.

$$
\tau=K \cdot\left(\Gamma^{0}+\Gamma\right)^{n}
$$

The interpolation is included in the matrix $\mathrm{C}$ of Equation 1 and is based on the following concepts. We assume 5 directions: $\underline{\mathrm{u}}_{\mathrm{i}}(\mathrm{i}=1 \ldots 5)$ advisedly chosen in the deviatoric strain rate space and the associated deviatoric stress: $\underline{\sigma}_{i}(i=1 \ldots 5)$ lying on the yield surface according to the Taylor model. These points will define the interpolation domain and will be called stress nodes. Additionally, we compute the contravariant vectors $\underline{S S}_{\mathrm{i}}$ and $\underline{u \underline{u}}_{\mathrm{i}}$ defined by Equations 3 and 4 .

$$
\begin{aligned}
& \underline{S S_{i}} \bullet \underline{\sigma_{j}}=\delta_{i j} \\
& \underline{u u_{i}} \bullet \underline{u_{j}}=\delta_{i j}
\end{aligned}
$$


With the use of those contravariant vectors we define intrinsic co-ordinates in the interpolation domain for any stress vector $\underline{\sigma}^{*}$ by projection according to Equation 5 and for any plastic strain rate direction $\underline{\mathrm{u}}^{*}$ with Equation 6.

$$
\begin{aligned}
& \eta_{i}=\underline{\sigma^{*}} \cdot \underline{s s_{i}} \\
& \eta_{i}=\underline{u^{*}} \bullet \underline{u u_{i}}
\end{aligned}
$$

The most important property of our stress-strain interpolation states that if the stress $\underline{\sigma}^{*}$ and the plastic strain rate direction $\underline{\mathrm{u}}^{*}$ physically correspond to the same point, then the intrinsic co-ordinates $\eta_{\mathrm{i}}$ computed for $\underline{\sigma}^{*}$ (Eqn. 5) or for $\underline{\underline{u}}^{*}$ (Eqn. 6) are equal.

The interpolation is achieved with the use of those intrinsic co-ordinates to compute the stress or the strain rate with a common formulation:

$$
\begin{aligned}
& \underline{\sigma}=\sum_{i} \eta_{i} \cdot \underline{\sigma_{i}} \\
& \underline{u}=\sum_{i} \eta_{i} \cdot \underline{u_{i}}
\end{aligned}
$$

Putting together Equations 6 and 7, we can compute the stress associated to a plastic strain rate direction and get the expression of the interpolation matrix $\underline{\underline{\mathrm{C}}}$ (the hardening not being taken into account here):

$$
\underline{\sigma}=\underbrace{\sum_{i} \underline{\sigma_{i}} \cdot \underline{u u_{i}}}_{=\underline{\underline{C}}} \bullet \underline{u}
$$

As long as the interpolation is achieved in the domain delimited by the 5 stress nodes, all the $5 \eta_{i}$ must remain between 0 and 1 . When one of the $5 \eta_{i}$ becomes negative, it means that the current stress is out of the domain and an updating of the stress nodes must take place. The classical updating method consists in finding 5 new stress nodes defining a new domain containing the current stress direction. The developed improved updating method makes use of the adjacent domain. Therefore, only 1 new stress node is computed with the Taylor model and 4 of the 5 old stress nodes are kept for the interpolation. The main advantages of this method are that it requires only 1 (instead of 5) Taylor model call for an updating and it improves the continuity of the resulting yield locus and the continuity of its normal.

The above considerations are sufficient to understand the interpolation approach that has finally been implemented in LAGAMINE code. However, it is interesting to note that further details and properties of such parameterisation of a $\mathrm{N}$ dimensional space were further investigated by [1] and [3]. They studied different interpolation methods on the interpolation domain: linear interpolation in Cartesian coordinates or hyperplane model, linear interpolation in spherical coordinates, approach enriched by bubble mode...

\subsection{Stress integration scheme}

As already mentioned, the stress-strain interpolation relation (Eqn. 1 and 9) does not use the concept of yield locus in a classical way. So, a specific integration scheme has been developed. The stress integration scheme implemented with our interpolation method is completely different from the classical radial return with elastic predictor; the main ideas are summarised in the flowchart of Figure 1 where obviously, no yield locus formulation is used. 


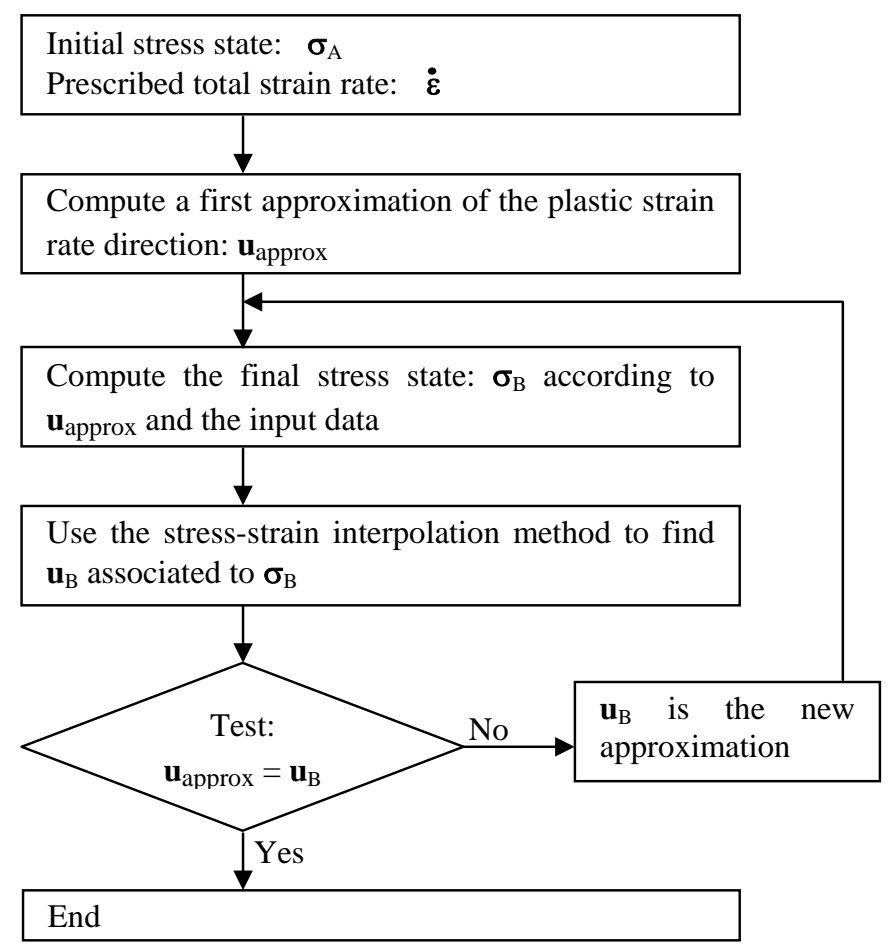

Figure 1: Stress integration scheme

As it has been observed during several finite element simulations, this stress integration scheme is well adapted for a local yield locus description and induces a reasonable number of interpolation domain updating.

\subsection{Implementation of the texture updating}

In this model, not only is the texture used to predict the plastic behaviour of the material, but also the strain history of each integration point is taken into account in order to update the texture.

The main ideas of the implementation are summarised in Figure 2. It should be noticed that the constitutive law in the FEM code is based on the interpolation method described earlier and on Taylor's model applied on the actual set of crystallographic orientations through the yield locus. These crystallographic orientations are represented with the help of the Euler angles ranging from $0^{\circ}$ to $360^{\circ}$ for $\varphi_{1}$ and from $0^{\circ}$ to $90^{\circ}$ for $\phi$ and $\varphi_{2}$ so as to take crystal cubic symmetry into account but not the sample symmetry. As shown on Figure 2, the texture updating is achieved outside the main part of the FEM code, for each interpolation point.

During a large finite element simulation, it is not reasonable to achieve a texture updating for each finite element and at the end of each time step. That is the reason why an updating criterion must be used to reduce computation time. This is still under investigation. At this stage, an updating occurs after a predefined number of time steps. A criterion based on a maximum cumulated plastic strain will also be examined.

The lattice rotation of each crystal, inducing the texture updating, is computed with Taylor's model by subtracting the slip induced spin from the rigid body rotation included in the strain history. 


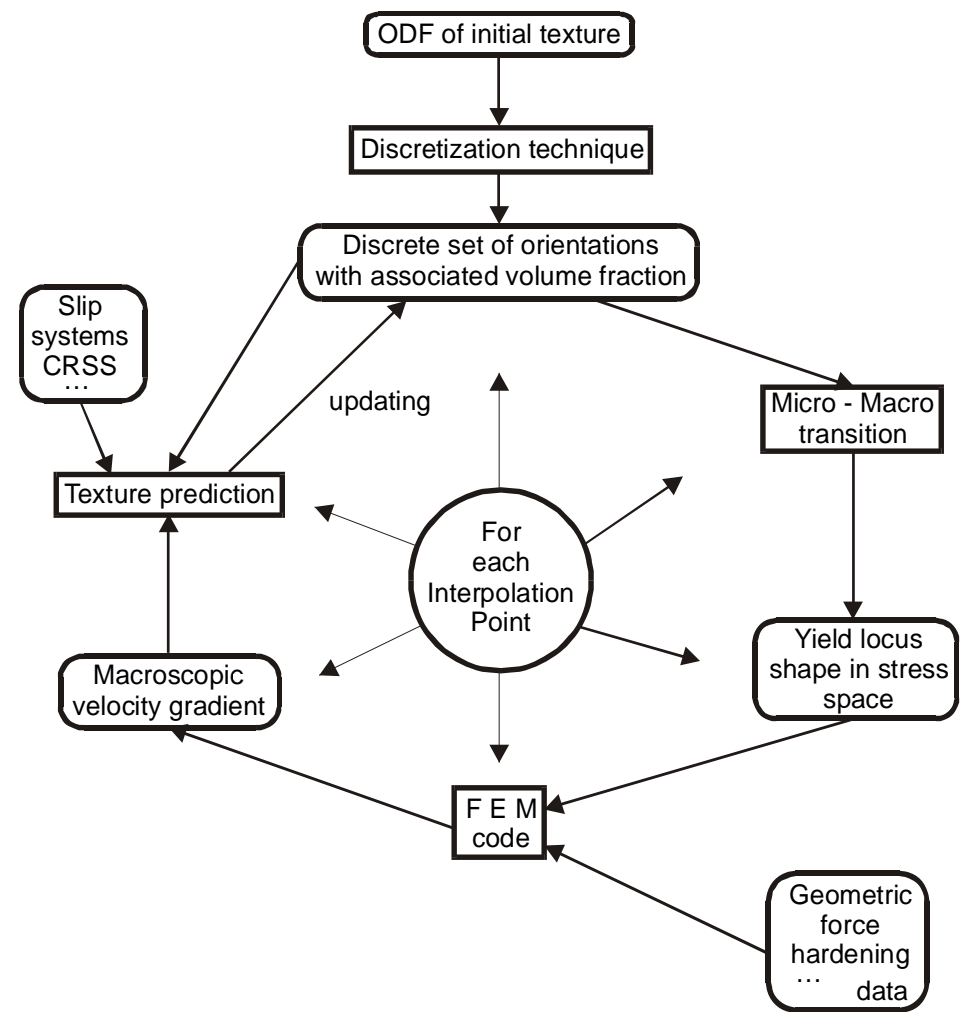

Figure 2: Coupling scheme for texture updating.

\section{DEEP-DRAWING SIMULATIONS}

In order to show up the influence of the texture evolution during a forming process, a deep-drawing simulation has been studied. The used steel is a high strength steel SPXI 250. The numerical data for this steel, i.e. the hardening behaviour, the Lankford coefficients,... are obtained from simple tensile tests. As we focus on the texture, the shape of the yield locus is deduced from its Orientation Distribution Function (ODF), which has been measured by X-ray diffraction.

The deep-drawing simulation is achieved with different constitutive laws. The stress-strain interpolation method described in section 2 is used. First without texture updating; so the representative set of orientations remains constant during the simulation. This model is referred to as 'S-s I' on the figure. Secondly, the texture is updated on the basis of the strain history of each finite element ('S-s I Evol' on the figure). Another texture based law is used: the $6^{\text {th }}$ order series yield locus described by [5]. This model is referred to as '6th order'. Finally we have achieved the deep-drawing simulation with a classical Hill 1948 constitutive law. The 6 parameters of the Hill law are fitted on simple tensile test experiments. They can be adjusted either on the yield stresses along 3 particular directions $\left(0^{\circ}, 45^{\circ}, 90^{\circ}\right.$ from the Rolling Direction in our case) or on the Lankford coefficients along the same directions. A mixed fitting can also be defined:

$$
\mathcal{H}^{\text {mixed }}=\vartheta \cdot \mathcal{H}^{r}+(1-\vartheta) \mathcal{H}^{\text {sig }}
$$

where $-\mathcal{H}^{r}$ represents the Hill parameters fitted on the Lankford coefficients,

$-\mathcal{H}^{\text {sig }}$

- $\mathcal{H}^{\text {mixed }}$ represents the mixed Hill parameters,

- $\vartheta$ is the fitting parameter which we have chosen to be equal to 1 ('Hill (r)' on the figure) and to 0.5 ('Hill $($ sig $+r)$ '). 


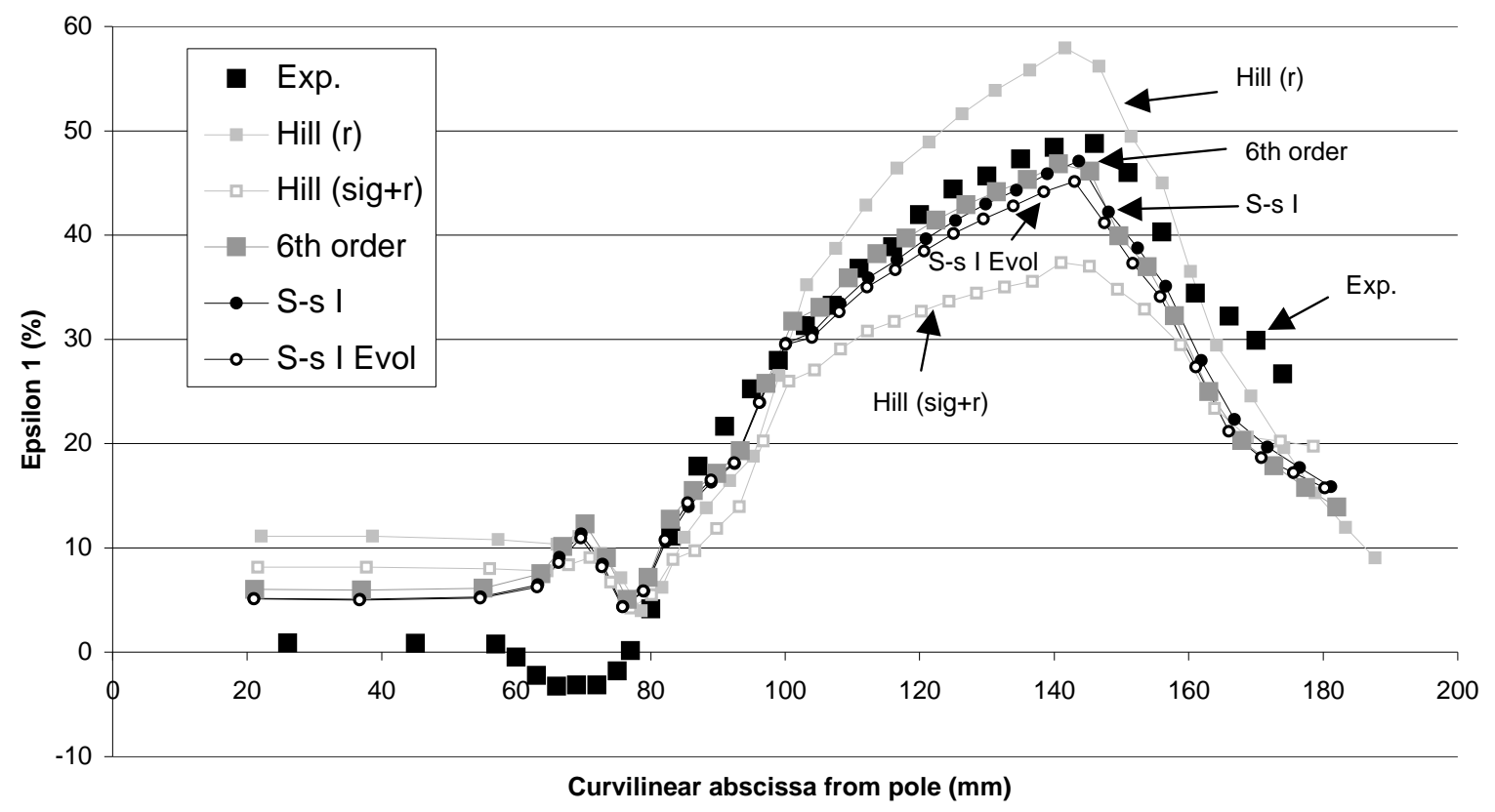

Figure 3: First principal strain along the transverse direction ; steel SPXI 250 ; Oil lubricant

Experimental results and measurements are used for the validation of these models.

Now, the geometry of the deep-drawing process should be presented. A $100 \mathrm{~mm}$ long cylindrical punch with a diameter of $150 \mathrm{~mm}$ and a curvature radius of $10 \mathrm{~mm}$, a die with a curvature radius of 10 $\mathrm{mm}$ and a blankholder are the drawing tools. The drawing ratio is 2.0; the blankholder force is $98.1 \mathrm{kN}$; the simulation is achieved up to a drawing depth of $80 \mathrm{~mm}$. The blank is a $0.8 \mathrm{~mm}$ thick SPXI250 steel. An oil is used for lubrication; a Coulomb law is modelling the friction with a coefficient of 0.13 .

For the numerical models described here above and for experimental results, the maximum principal strain distribution on a section along the transverse direction is reported on Figure 3. It should be noticed that the strain is relatively small at the top of the cup (near the pole; for s smaller than $80 \mathrm{~mm}$ ) while large displacements take place. Indeed, in that part of the cup, the steel sheet is applied against the punch and follows the punch travel without large deformation. Then, at the flange of the cup, the maximum principal strain suddenly increases and is maximum for s being equal to $145 \mathrm{~mm}$. This maximum is located at the vertical part of the flange where the cup is free of contact with the punch and the matrix. After the maximum, the principal strain $\varepsilon_{1}$ decreases as the contact with the matrix reduces the tension of the sheet. The resulting maximum principal strain obtained with Hill law is quite too high or too low depending on the fitting used, while texture based laws are in agreement with experimental results.

The punch force as a function of the punch travel is presented on Figure 4. These curves are not linked to the anisotropy of the steel sheet but to the global stiffness of the material and then to the hardening behaviour. It can be noticed that the curve with the Hill law fitted on the Lankford coefficients is a little bit too high; Hill with a mixed fitting is in agreement with experimental results. All the texture based laws are superimposed and their predicted maximum punch force is too low.

Finally, Figure 5 is directly linked to the anisotropy of the steel sheet. For finite element simulations, this anisotropy is induced by the constitutive law (either from the Hill coefficients or the texture data). The Lankford coefficients used for the constitutive law based on the stress-strain interpolation with a constant texture come from the black thick curve. Two sets of experimentally measured Lankford coefficients are also presented. Furthermore, on the finite element mesh, 3 particular elements are chosen along the 3 studied directions $\left(0^{\circ}, 45^{\circ}\right.$ and $90^{\circ}$ from Rolling Direction) such that they undergo completely the drawing process on the curvature of the die; they are located at mid-height on the flank of the deformed cup. The texture at the end of the deep-drawing process for the law using an evolving texture is examined for these three elements. As the Lankford coefficient has proved to be an important parameter for deep-drawing processes, it is computed for the 3 final textures. Significant differences of the $r$ values 
appear for the 3 particular directions according to the initial curve and to each other.

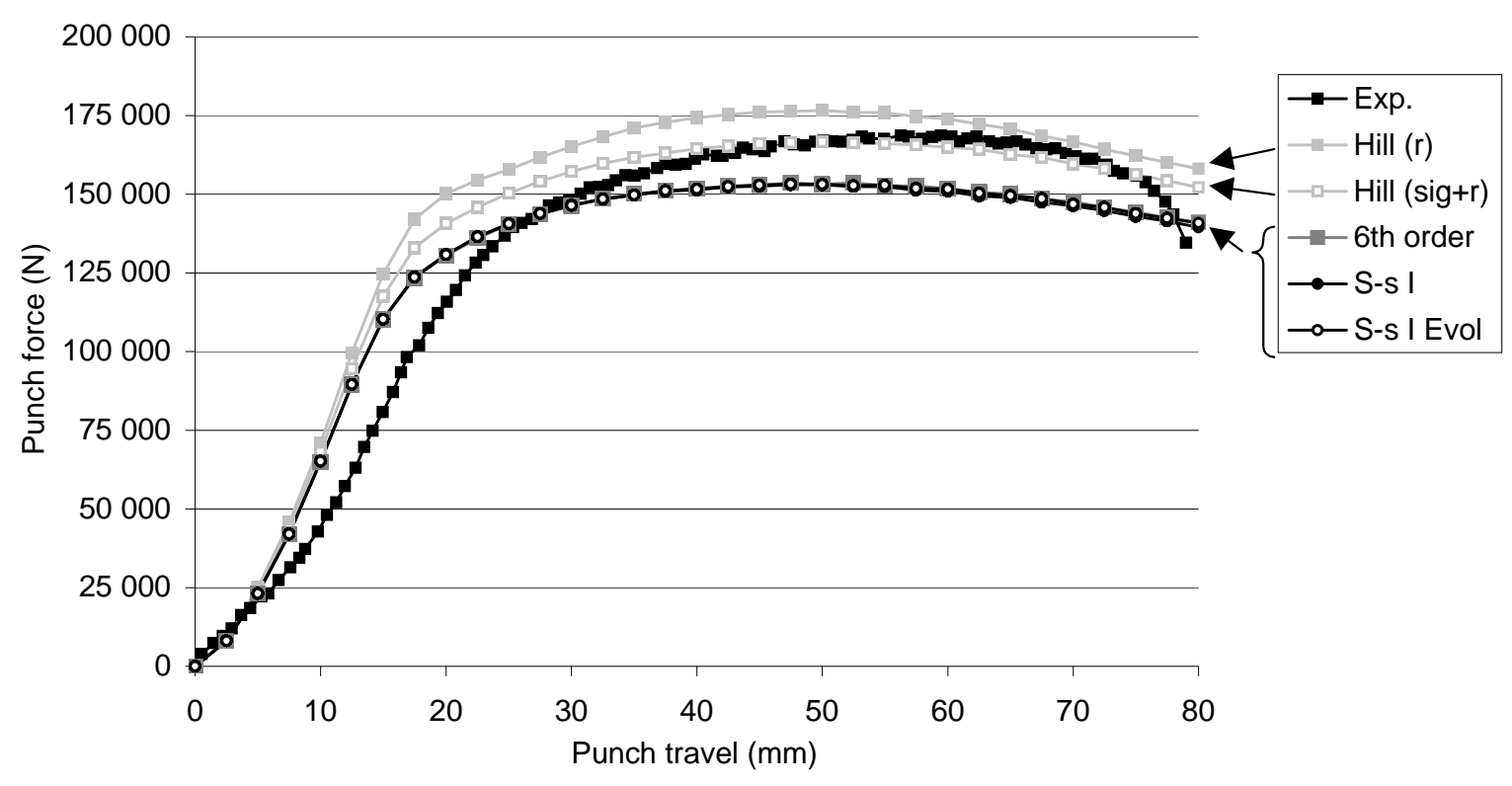

Figure 4: Punch force versus punch travel

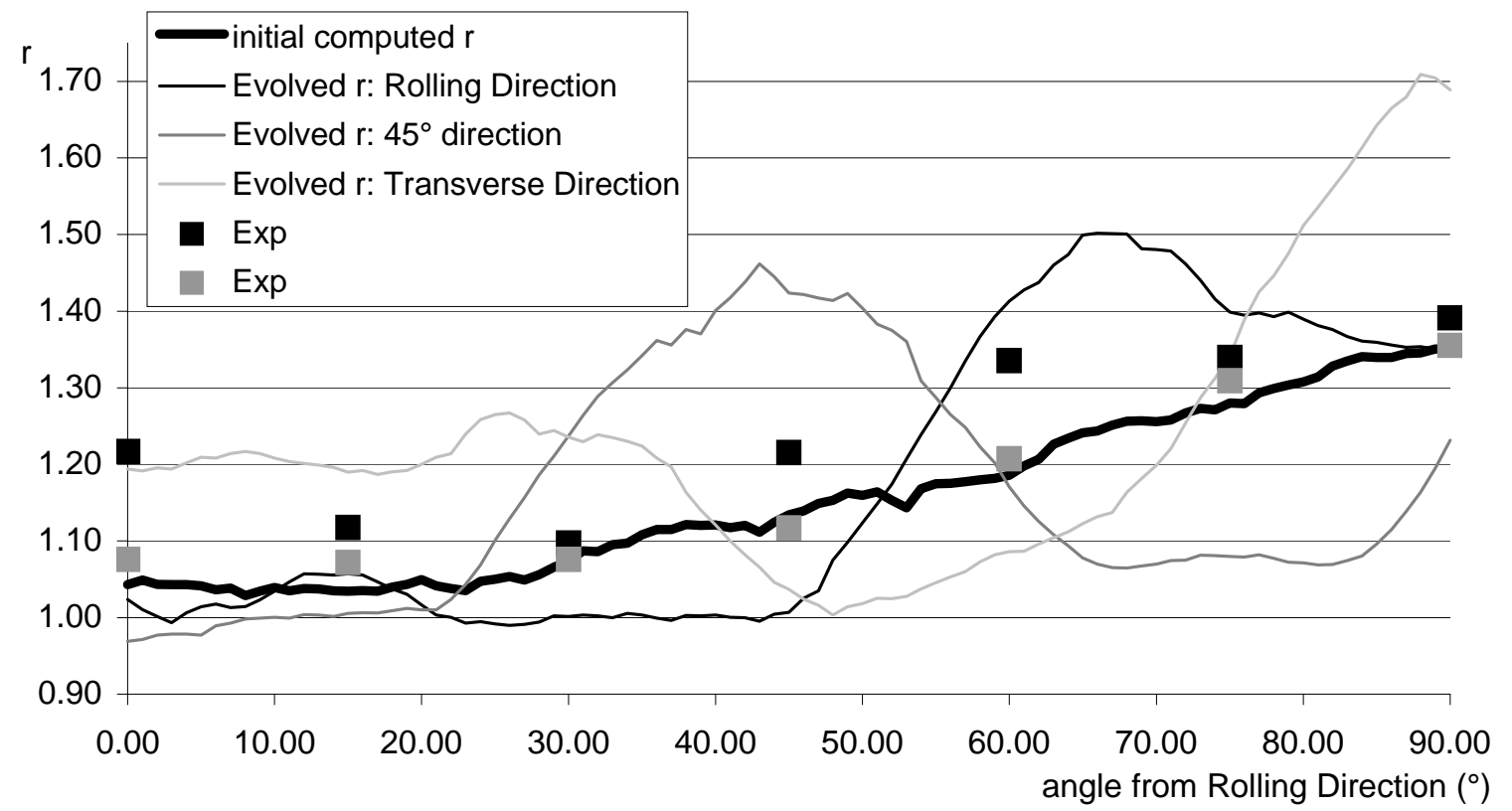

Figure 5: Lankford coefficient before and after deep-drawing (final textures are taken at mid-height of the blank's flank) 


\section{CONCLUSION}

On the deep-drawing application presented here, the influence of the evolving texture is relatively small on the main results of the simulation. However, if we focus on the final texture and particularly on the resulting Lankford coefficients, a large difference can be noticed from one element to another and to the initial behaviour. It is then important to take into account the texture evolution of the steel sheet for each element of the mesh. This leads to computing problems: high need in memory storage and large computation time. Nevertheless, parallel computation allows us to manage such finite element simulations within sensible delays.

\section{Acknowledgements}

As Research Associate of the National Fund for Scientific Research (Belgium), A. M. Habraken would like to thank this Belgian research fund for its support.

\section{References}

[1] Duchêne L., "Implementation of a yield locus interpolation method in the finite element code LAGAMINE”, DEA Graduation Work, Ulg, Liège, Belgium (2000).

[2] Duchêne L., Godinas A. and Habraken A. M., "Metal plastic behaviour linked to texture analysis and FEM method”, Proc. $4^{\text {th }}$ Int. Conf.: NUMISHEET’99, pp. 97-102.

[3] Godinas A., "Définition locale du comportement plastique d'un matériau”, Intermediate report n²7, Convention RW n²748, Région Wallonne, Ulg, Liège, Belgium (1998).

[4] Munhoven S., Habraken A. M., Van Bael A. and Winters J., "Anisotropic finite element analysis based on texture”, Proc. $3^{\text {rd }}$ Int. Conf.: NUMISHEET’96, pp. 112-119.

[5] Winters J., "Implementation of Texture-Based Yield Locus into an Elastoplastic Finite Element Code”, Ph. D. Thesis, KUL, Leuven, Belgium (1996). 